\title{
Condiciones de vida, estado nutricional y estado de salud en adultos mayores, Córdoba, Argentina
}

\author{
Life conditions, nutritional and health status in elderly population, Córdoba, Argentina
}

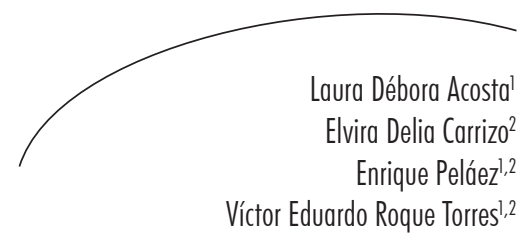

\section{Resumen}

Objetivo: Describir y comparar el estado nutricional y el estado de salud en dos grupos de adultos mayores de diferentes condiciones de vida, residentes en la ciudad de Córdoba, Argentina. Material y métodos: Estudio transversal, llevado a cabo en dos grupos: adultos mayores concurrentes a Comedores Sociales de la Provincia de Córdoba (CSPC; $\mathrm{n}=308$ ) y adultos mayores afiliados a la obra social universitaria Dirección de Asistencia Social del Personal Universitario (DASPU; $n=134$ ). Se determinaron parámetros antropométricos: índice de masa corporal (IMC), circunferencia de cintura (CC) e índice cintura cadera (ICC). Para la determinación del estado de salud se utilizaron indicadores subjetivos (autopercepción de salud, autoinforme de enfermedades crónicas), obtenidos a través de encuesta. Para la corroboración de hipótesis se utilizó Test de X2, cálculo de Odds Ratio (OR), y Prueba T. Resultados: En hombres, se observó mayor sobrepeso y obesidad en el grupo DASPU; mientras que en las mujeres no se encontraron mayores diferencias entre ambas instituciones, salvo un promedio menor de IMC en el grupo DASPU. En relación a los indicadores del estado de salud, se observó en ambos sexos, una mejor percepción de la salud en el grupo DASPU, mayor prevalencia de diabetes en hombres del grupo CSPC, hipertensión en mujeres de CSPC y tumor maligno en hombres del grupo DASPU. Conclusiones: Se observa que las condiciones sociales de vida influyen en el estado nutricional y de salud de los adultos mayores, en especial en hombres. Esto implica riesgos diferentes para ambos grupos.

\footnotetext{
Universidad Nacional de Córdoba. Centro de Investigaciones y Estudios sobre Cultura y Sociedad, Unidad Ejecutora del Consejo Nacional de Investigaciones Científicas y Técnicas. Córdoba, Argentina.

2 Universidad Nacional de Córdoba. Facultad de Ciencias Económicas. Córdoba, Argentina.
}

Financiamento: Proyecto subsidiado por la Secretaria de Ciencia y Tecnología de la Universidad Nacional de Córdoba, Argentina. Convocatoria 2010-2011. Resolución 214/2010

Correspondência / Correspondence

Laura Débora Acosta

E-mail: laudeac@hotmail.com
Palabras claves: Adulto Mayor. Anciano. Salud. Nutrición. Condiciones Sociales. 


\section{Abstract}

Objective: To describe and compare nutritional and health status in two groups of elderly population with different life conditions, living in Córdoba city, Argentina. Material and methods: Cross-sectional study in two samples of elderly: aged people of Social Food Program Beneficiary, in Córdoba city (SFPB), $n=308$ ); and aged people affiliated to University Personal Health Insurance (UPHI; n=134). Anthropometric measures were applied: body mass index (BMI), waist circumference (WC) and waisthip ratio (WHR). Health status was determinate with subjective indicators (self health perception, chronic conditions self report), measure through questionnaire. Statistical analysis included Chi-Square test, Odds Ratio (OR) and T test. Results: In men, we observed more overweight and obesity in UPHI group than in SFPB; while in women not differences was find in two groups, excepting a lower mean of BMI in UPHI group. Health indicators show in both sexes a better self-perception health in UPHI group. We observed more diabetes prevalence in men of SFPB group, more hypertension in women of SFPB group, and more malign tumor in men of UPHI group. Conclusions: We observed that social conditions had influence in nutritional and health status of elderly people, especially in men. For this reason, life conditions conducted to different risks in these groups if elderly people.
Key words: Elderly. Health. Nutrition. Nutritional Status. Social Conditions.

\section{INTRODUCCIÓN}

El proceso de envejecimiento de la población se produce de diferentes maneras en las distintas regiones del mundo: en la población latinoamericana dicho fenómeno ocurre de una manera más acelerada que en los países del mundo desarrollado ${ }^{1}$ ya que mientras en Europa este proceso demoró entre 150 y 200 años, en Latinoamérica el mismo fenómeno se desarrolló sólo en 40 y 60 años, sumado al hecho de ser una sociedad empobrecida con graves desigualdades en la distribución del ingreso.

Los países de América Latina, para enfrentar este proceso de envejecimiento poblacional, deben conocer los factores que afectan a la población adulta, no sólo por la situación presente si no también de cara al futuro que se vislumbra: como consecuencia de la dinámica demográfica la proporción de personas mayores se triplicará entre los años 2000 a 2050. Para esta última fecha las proyecciones señalan que uno de cada cuatro latinoamericanos será una persona adulta mayor. ${ }^{2}$
Así, el aumento de población mayor a 60 años en las últimas décadas ha generado que se indague acerca de los factores que afectan su autonomía y calidad de vida. Dentro de éstos factores se destacan los problemas nutricionales ${ }^{3,4}$ las enfermedades crónicas 5,6 y las condiciones de vida., ${ }^{5,-9}$

En relación a los problemas nutricionales en los adultos mayores de la región de América Latina, se subraya que los cambios en el estilo de vida y la urbanización han influido en el proceso denominado "transición nutricional", el cual acompaña el proceso de transición epidemiológica y demográfica. La transición nutricional es un fenómeno caracterizado por condiciones de deficiencia nutricional, típicas de sociedades en desarrollo; acompañado por una alta prevalencia de malnutrición por exceso - y consecuentemente un aumento de la prevalencia de enfermedades crónicas no transmisibles-, propias de sociedades modernas, que coexisten en comunidades de bajos recursos. ${ }^{10,11}$

Según la encuesta Salud Bienestar y Envejecimiento (SABE) en América Latina y el Caribe $^{12}$ en el año 2000, la proporción de 
individuos en edad mayor que presentan obesidad varía entre el 35\% en Montevideo al 20\% en Sao Paulo, mientras que la proporción de personas mayores con bajo peso varía del 15\% en la ciudad de México al 29\% en Bridgetown.

En Argentina, según datos de la Encuesta Nacional de Factores de Riesgo 2005, el 17,7\% de los adultos mayores de 65 años y más presentaron obesidad. ${ }^{13}$ En cuanto a la prevalencia de enfermedades crónicas, el 47,1\% de la población adulta mayor presentó hipertensión arterial, el 38\% presenta colesterol elevado y el 20,4\% presentan glucemia elevada. ${ }^{14}$ Existe además un mayor riesgo de padecer algunas patologías crónicas y obesidad a menores niveles de ingresos y de escolarización. ${ }^{15}$

De este modo, el presente trabajo tuvo como propósito describir y comparar el estado nutricional y el estado de salud en dos grupos de adultos mayores de diferentes condiciones de vida, residentes en la ciudad de Córdoba, Argentina; durante el año 2011.

\section{MATERIAL Y MÉTODOS}

Se utilizaron datos primarios provenientes de la investigación "Condiciones de Vida y Salud Nutricional en los Adultos Mayores de la ciudad de Córdoba". Se trata de un estudio descriptivo y correlacional, cuya población está compuesta por personas de 60 años y más, autoválidos y residentes en la ciudad de Córdoba, Argentina; encuestados durante el año 2011. Se seleccionaron dos muestras probabilísticas provenientes de diferentes poblaciones de adultos mayores: La primera está constituida por 308 adultos mayores concurrentes a Comedores Sociales de la Provincia de Córdoba (CSPC; $\mathrm{n}=8.654)$ y 134 adultos mayores afiliados a la obra social del personal Docente y No Docente de la Universidad Nacional de Córdoba: Dirección de Asistencia Social del Personal Universitario (DASPU; $\mathrm{n}=1.236$ ).

La muestras seleccionadas en el presente trabajo fueron intencionales, con la finalidad de disponer de datos de dos grupos poblacionales de condiciones socioeconómicas diferentes: uno con un nivel socioeconómico medio y medioalto, caracterizado por poseer un alto porcentaje de estudios superiores y otro grupo de adultos mayores de niveles medio-bajo y bajo, tanto en los ingresos como en nivel de escolarización.

El proyecto fue aprobado por el Comité de Ética de la Facultad de Ciencias Médicas de la Universidad Nacional de Córdoba, resolución $n^{\circ}$ 023/10. Se solicitó a cada encuestado la firma del consentimiento informado. No contar con el consentimiento del adulto mayor, constituyó el único criterio de exclusión para participar en este estudio.

Para la determinación del Estado Nutricional se utilizaron técnicas medicionales (antropometría) lo cual incluyó el Índice de Masa Corporal (IMC),16 Circunferencia de Cintura (CC) e Índice Cintura Cadera (ICC).

El IMC se obtuvo a partir de la ecuación peso/talla,2 se categorizó según lo planteando por la Organización Panamericana de la Salud (OPS)17 para las personas adultas mayores, estableciendo como valores de "normalidad" un IMC de $23-28 \mathrm{~kg} / \mathrm{m} 2$, "malnutrición por déficit" $<23 \mathrm{~kg} / \mathrm{m} 2$ y "malnutrición por exceso" $>28 \mathrm{~kg} /$ $\mathrm{m} 2$. El peso fue medido con Balanza Electrónica "Tanita", con precisión de 100 gramos; y la talla fue estimada mediante la altura de la rodilla, la cual es medida con antropómetro largo, con precisión en milímetros. Para la estimación de la talla se aplica la ecuación de Chumlea. ${ }^{17,18}$ Además se calculó la talla en bipedestación, para lo cual se utilizó estadiómetro con precisión en milímetros. Se calculó en IMC con talla estimada y talla en bipedestación.

Las categorías de circunferencia de cintura consideradas fueron "sin riesgo" (hasta 87,99 cm en el caso de mujeres y hasta $101,99 \mathrm{~cm}$ en hombres) y "con riesgo" ( $88,00 \mathrm{~cm}$ o más para las mujeres y mayor o igual $102,00 \mathrm{~cm}$ en hombres). Para el índice cintura cadera - obtenido del cociente entre la circunferencia de la cintura en relación a la circunferencia de la cadera- se 
consideró "riesgo" cuando el valor del mismo fue mayor o igual que 1 para hombres y mayor o igual que 0,80 en mujeres. Las circunferencias de cintura y cadera fueron medidas con cinta de fibra de vidrio inextensible y flexible, con precisión en milímetros. Las mediciones fueron realizadas por Lic. en nutrición y estudiantes de nutrición entrenados para tal fin. Todas las mediciones se tomaron por triplicado y luego se determinó el promedio de las mismas.

Por otra parte, para la determinación del estado de salud se utilizaron indicadores obtenidos a través de las respuestas del formulario de la encuesta, lo cual incluyó la percepción del estado de salud - la cual incluye las categorías: "excelente", "muy bueno", "bueno", "regular" y "malo"-. Además, se utilizó el autoinforme de presencia de las siguientes condiciones crónicas: hipertensión arterial; diabetes; tumores malignos; enfermedad del corazón; accidente cerebro vascular; artritis, artrosis o reumatismo. Los cuestionarios fueron administrados por encuestadores entrenados.

La información fue procesada con el paquete estadístico SPSS versión 11.5 para Windows. Se determinaron frecuencias absolutas y relativas expresadas en porcentajes; medias y desviación estándar (DE), según la naturaleza de la variable analizada. Para la corroboración de hipótesis seutilizó Test de X2 , Test de Fisher; cálculo de Odds Ratio $(\mathrm{OR})$, y Prueba T, con sus respectivos intervalos de confianza (IC) aceptándose un nivel de significación inferior a 0,05 para rechazar de hipótesis nula. RESULTADOS

El 62\% de los encuestados en el grupo de CSPC correspondieron al sexo femenino, mientras que en el grupo DASPU el 61,2\% fueron mujeres. La media de edad fue de 70 años DE $\pm 7,9$ en DASPU y de 70,9 años en CSPC DE $\pm 7,0$. En cuanto al nivel de instrucción, se percibe una importante disparidad entre ambos grupos: el 72,9\% de la población de adultos mayores de DASPU posee secundario completo o estudios universitarios o terciarios, mientras que el $8,9 \%$ de los encuestados en el grupo CSPC posee secundario completo o más. Asimismo hay diferencias en el ingreso total familiar percibido: mientras el $82,6 \%$ de los encuestados en CSPC percibe ingresos mensuales inferiores a los USD 500 (de los cuales el 77,0\% es inferior a los USD 250); sólo el 19,4\% de los adultos mayores de DASPU percibe ingresos entre USD 250 y USD 500; y el resto recibe ingresos superiores a los USD 500.

En cuanto a la los indicadores antropométricos de los varones, se observa una media superior en el peso en el grupo DASPU, como así también valores superiores en la talla en bipedestación, la circunferencia de cintura, circunferencia de cadera y de IMC según talla en bipedestación. Pero, por otra parte, se observó una mayor media en la talla estimada en la población de CSPC en relación con el grupo DASPU (tabla 1). 
Tabla 1. Estadísticos descriptivos de indicadores antropométricos. Adultos mayores de 60 años y más, sexo masculino; obra social DASPU y concurrentes a CSPC. Córdoba, Argentina, 2011.

\begin{tabular}{|c|c|c|c|c|c|c|c|c|c|c|}
\hline \multirow[b]{2}{*}{ Indicador } & \multirow[b]{2}{*}{ Mínimo } & \multicolumn{2}{|l|}{ CSPC } & \multicolumn{4}{|c|}{ DASPU } & \multirow[b]{2}{*}{ DE } & \multirow[b]{2}{*}{ Sig. } & \multirow{2}{*}{$\begin{array}{c}\text { Dif. Medias } \\
\text { (IC 95\%) }\end{array}$} \\
\hline & & Máximo & Media & $\mathrm{DE}$ & Mínimo & Máximo & Media & & & \\
\hline & & & & & & & & & & $-6,3$ \\
\hline \multirow[t]{2}{*}{ Peso } & 50,6 & 111,2 & 75,4 & 13,0 & 58,4 & 108,7 & 81,7 & 11,1 & 0,004 & $(-10,5)-(-2,1)$ \\
\hline & & & & & & & & & & 5,3 \\
\hline \multirow[t]{2}{*}{ Talla estimada } & 153,0 & 192,0 & 175,2 & 7,9 & 149,7 & 183,0 & 169,9 & 5,8 & $<0,001$ & $3,1-7,5$ \\
\hline & & & & & & & & & & $-4,3$ \\
\hline \multirow[t]{2}{*}{ Talla en bipedestación } & 149,5 & 185,0 & 166,6 & 6,9 & 157,0 & 187,5 & 170,9 & 5,8 & $<0,001$ & $(-6,5)-(-2,8)$ \\
\hline & & & & & & & & & & $-3,6$ \\
\hline \multirow[t]{2}{*}{ Circunferencia de cintura } & 66,5 & 133,0 & 95,9 & 10,4 & 76,0 & 122,5 & 99,6 & 9,9 & 0,039 & $(-7,0)-(1,8)$ \\
\hline & & & & & & & & & & $-2,8$ \\
\hline \multirow[t]{2}{*}{ Circunferencia cadera } & 85,0 & 138,0 & 102,4 & 8,7 & 96,0 & 120,0 & 105,2 & 6,0 & 0,042 & $(-5,4)-(-0,1)$ \\
\hline & & & & & & & & & & $-3,8$ \\
\hline \multirow[t]{2}{*}{ IMC (talla estimada) } & 17,1 & 34,7 & 24,5 & 3,8 & 21,2 & 42,4 & 28,3 & 4,1 & $<0,001$ & $(-5,1)-(-2,5)$ \\
\hline & & & & & & & & & & $-0,82$ \\
\hline \multirow[t]{2}{*}{ IMC (talla bipedestación) } & 17,9 & 40,6 & 27,1 & 3,9 & 20,2 & 37,4 & 27,9 & 3,8 & 0,213 & $(-2,1)-(0,5)$ \\
\hline & & & & & & & & & & $-0,01$ \\
\hline ICC & 0,76 & 1,10 & 0,94 & 0,06 & 0,77 & 1,07 & 0,95 & 0,06 & 0,414 & $0,01-(-0,02)$ \\
\hline
\end{tabular}

En el caso de las mujeres, se observan diferencias en la media de la talla en bipedestación, siendo mayor en el grupo DASPU. Por otra parte, existió una mayor circunferencia de cadera en el grupo CSPC y un mayor IMC obtenido a través de la talla en bipedestación (tabla 2). 
Tabla 2. Estadísticos descriptivos de indicadores antropométricos. Adultos mayores de 60 años y más, sexo masculino; obra social DASPU y concurrentes a CSPC. Córdoba, Argentina, 2011.

\begin{tabular}{|c|c|c|c|c|c|c|c|c|c|c|}
\hline \multirow[b]{2}{*}{ Indicador } & \multirow[b]{2}{*}{ Mínimo } & \multicolumn{2}{|l|}{ CSPC } & \multicolumn{4}{|c|}{ DASPU } & \multirow[b]{2}{*}{ DE } & \multirow[b]{2}{*}{ Sig. } & \multirow{2}{*}{$\begin{array}{l}\text { Dif. Medias } \\
\text { (IC 95\%) }\end{array}$} \\
\hline & & Máximo & Media & $\mathrm{DE}$ & Mínimo & Máximo & Media & & & \\
\hline & & & & & & & & & & 2,1 \\
\hline \multirow[t]{2}{*}{ Peso } & 40,2 & 110,1 & 69,4 & 14,5 & 43,1 & 95,5 & 67,2 & 11,2 & 0,195 & $(-1,1)-(5,5)$ \\
\hline & & & & & & & & & & $-0,39$ \\
\hline \multirow[t]{2}{*}{ Talla estimada } & 140,0 & 181,0 & 157,5 & 5,9 & 142,0 & 176,0 & 157,9 & 5,3 & 0,619 & $(-1,9)-(1,2)$ \\
\hline & & & & & & & & & & $-3,4$ \\
\hline \multirow[t]{2}{*}{ Talla en bipedestación } & 139,5 & 169,0 & 153,6 & 63 & 141,5 & 195,0 & 156,9 & 7,5 & $<0,001$ & $(-5,1)-(1,6)$ \\
\hline & & & & & & & & & & 2,5 \\
\hline \multirow[t]{2}{*}{ Circunferencia de cintura } & 62,5 & 128,4 & 91,9 & 13,1 & 66,0 & 114,0 & 89,3 & 10,5 & 0,105 & $(-0,5)-(5,6)$ \\
\hline & & & & & & & & & & 3,2 \\
\hline \multirow[t]{2}{*}{ Circunferencia cadera } & 83,0 & 141,0 & 107,5 & 11,9 & 89,0 & 128,0 & 104,3 & 8,9 & 0,021 & $0,5-5,8$ \\
\hline & & & & & & & & & & 0,9 \\
\hline \multirow[t]{2}{*}{ IMC (talla estimada) } & 16,3 & 46,9 & 27,9 & 5,4 & 18,0 & 38,0 & 26,9 & 4,2 & 0,131 & $(-0,3)-(2,2)$ \\
\hline & & & & & & & & & & 1,9 \\
\hline \multirow[t]{2}{*}{ IMC (talla bipedestación) } & 16,5 & 43,7 & 29,5 & 5,9 & 14,4 & 38,5 & 27,5 & 4,3 & 0,004 & $0,6-3,2$ \\
\hline & & & & & & & & & & $-0,002$ \\
\hline ICC & 0,68 & 1,11 & 0,85 & 0,07 & 0,68 & 1,03 & 0,86 & 0,07 & 0,765 & $(-0,02)-(0,01)$ \\
\hline
\end{tabular}

$\mathrm{Al}$ analizar el estado nutricional en varones según IMC, obtenido a través de la talla estimada, se observan diferencias en la categoría "déficit", siendo mayor la proporción en los adultos mayores provenientes de los CSPC que presentan déficit en el estado nutricional. Se observa además una mayor proporción de "exceso" en el grupo DASPU. En el caso del estado nutricional obtenido a través del IMC según talla estimada en varones, no se observaron diferencias estadísticamente significativas (tabla 3). Respecto a la circunferencia de la cintura en varones, existió una mayor proporción de la categoría "riesgo" en el grupo DASPU. Sin embargo, no se observaron diferencias en el índice cintura/cadera (tabla 3). 
Tabla 3. Indicadores de estado nutricional, según sexo. Adultos mayores de 60 años y más; CSPC ( $\mathrm{n}=308)$ y obra social DASPU (n=134). Córdoba, Argentina, 2011.

\begin{tabular}{|c|c|c|c|c|c|c|c|c|c|}
\hline \multirow[t]{2}{*}{ Indicador } & \multirow[t]{2}{*}{ Categoría } & \multicolumn{2}{|c|}{ Masculino } & \multirow{2}{*}{$\begin{array}{l}\text { OR** }^{* *} \\
\text { (IC 95\%) }\end{array}$} & \multirow[t]{2}{*}{ Sig. } & \multicolumn{2}{|c|}{ Femenino } & \multirow{2}{*}{$\begin{array}{l}\mathrm{OR}^{* *} \\
\text { (IC 95\%) }\end{array}$} & \multirow[t]{2}{*}{ Sig. } \\
\hline & & CSPC & DASPU & & & CSPC & DASPU & & \\
\hline \multirow{6}{*}{$\begin{array}{l}\text { Estado } \\
\text { nutricional s/talla } \\
\text { estimada }\end{array}$} & Normal* & 50 & 22 & - & - & 70 & 29 & - & - \\
\hline & & $(44,6 \%)$ & $(44,9 \%)$ & & & $(38,3 \%)$ & $(39,2 \%)$ & & \\
\hline & Déficit & 40 & 2 & 8,8 & 0,01 & 34 & 15 & 0,9 & 0,86 \\
\hline & & & $(35,7 \%)$ & $(4,1 \%$ & $(1,9-39,7)$ & $(18,6 \%)$ & $(20,3 \%)$ & $(0,4-1,9)$ & \\
\hline & Exceso & 22 & 25 & 0,4 & 0,02 & 79 & 30 & 1,1 & 0,77 \\
\hline & & $(19,6 \%)$ & $(51,0 \%)$ & $(0,2-0,8)$ & & $(43,2 \%)$ & $(40,5 \%)$ & $(0,6-2,0)$ & \\
\hline \multirow{6}{*}{$\begin{array}{l}\text { Estado } \\
\text { nutricional s/talla } \\
\text { bipedestación }\end{array}$} & Normal* & 51 & 18 & - & - & 63 & 25 & - & \\
\hline & & $(44,7 \%)$ & $(37,5 \%)$ & & & $(34,2 \%)$ & $(34,2 \%)$ & & \\
\hline & Déficit & 14 & 6 & 8,2 & 0,82 & 22 & 11 & 0,8 & 0,59 \\
\hline & & $(12,3 \%)$ & $(12,5 \%)$ & $(0,3-2,5)$ & & $(12,0 \%)$ & $(15,1 \%)$ & $(0,3-1,9)$ & \\
\hline & Exceso & 49 & 24 & 0,7 & 0,38 & 99 & 37 & 1,1 & 0,84 \\
\hline & & $(43,0 \%)$ & $(50,0 \%)$ & $(0,3-1,5)$ & & $(53,8 \%)$ & $(50,7 \%)$ & $(0,6-1,9)$ & \\
\hline \multirow{4}{*}{$\begin{array}{l}\text { Circunferencia } \\
\text { de cintura }\end{array}$} & Sin riesgo* & 85 & 26 & - & - & 73 & 30 & - & - \\
\hline & & $(73,9 \%)$ & $(52,0 \%)$ & & & $(39,5 \%)$ & $(40,0 \%)$ & & \\
\hline & Riesgo & 30 & 24 & 26 & 0,01 & 112 & 45 & 0,9 & 0,52 \\
\hline & & $(26,1 \%)$ & $(48,0 \%)$ & $(1,3-5,2)$ & & $(60,5 \%)$ & $(60,0 \%)$ & $(0,6-1,7)$ & \\
\hline \multirow{4}{*}{$\begin{array}{l}\text { Índice } \\
\text { cintura/cadera }\end{array}$} & Sin riesgo* & 100 & 39 & - & - & 38 & 14 & - & - \\
\hline & & $(87,0 \%)$ & $(78,0 \%)$ & & & $(20,8 \%)$ & $(18,7 \%)$ & & \\
\hline & Riesgo & 15 & 11 & 1,8 & 0,1 & 145 & 61 & 1,1 & 0,42 \\
\hline & & $(13 \%)$ & $(22.0 \%)$ & $(0,8-4,5)$ & & $(79,2 \%)$ & $(81,3 \%)$ & $(0,6-2-3)$ & \\
\hline
\end{tabular}

${ }^{*}$ Categoría de referencia; $* * \mathrm{OR}=$ Odds ratio $\mathrm{CSPC}=$ Comedores Sociales de la Provincia de Córdoba; DASPU = Dirección de Asistencia Social del Personal Universitario.

En el caso de las mujeres, no se observan prácticamente diferencias en el estado nutricional determinado a través de la talla estimada y la talla en bipedestación, observándose en ambos grupos una mayor proporción de la categoría "exceso" (tabla 3). Lo mismo se observa en el caso de la circunferencia de la cintura y el índice cintura cadera, existiendo una alta proporción de la categoría "riesgo" en ambos indicadores tanto para el grupo CSPC como el grupo DASPU (tabla 3).

En relación al estado de salud, se observa en la tabla 4 el 59,8\% de la población de varones adultos mayores provenientes de CSPC indicó que su salud era "excelente", "muy buena" o "buena" mientras que un porcentaje mayor de adultos mayores del grupo DASPU (80,8\%) manifestaron una apreciación positiva de su salud $(p=0,01)$. En el caso de las mujeres del grupo CSPC 50,8\% manifestaron que su salud era "excelente, muy buena o buena", mientras que el 68,3\% de las mujeres de DASPU tuvieron una percepción positiva de su salud $(\mathrm{p}=0,006)$. Se advierte, de este modo, que tanto en hombres como mujeres los afiliados a DASPU presentan mejor autopercepción de salud que los concurrentes a los CSPC, siendo resultados estadísticamente significativos. 
Tabla 4. Estado de salud según indicadores subjetivos. Adultos mayores de 60 años y más, según sexo. CSPC ( $\mathrm{n}=308)$ y obra social DASPU ( $\mathrm{n}=134)$. Córdoba, Argentina, 2011.

\begin{tabular}{|c|c|c|c|c|c|}
\hline \multirow[t]{2}{*}{ Indicador } & \multirow[t]{2}{*}{ Categorías } & \multicolumn{2}{|l|}{ Masculino } & \multicolumn{2}{|l|}{ Femenino } \\
\hline & & CSPC & DASPU & CSPC & DASPU \\
\hline \multirow{7}{*}{$\begin{array}{l}\text { ¿Diría usted que su salud es excelente, } \\
\text { muy buena, buena, regular o mala? }\end{array}$} & Excelente & $8(6,8 \%)$ & $4(7,7 \%)$ & $10(5,2 \%)$ & $5(6,1 \%)$ \\
\hline & Muy buena & $14(12,0 \%)$ & $12(23,1 \%)$ & $25(13,1 \%)$ & $17(20,7 \%)$ \\
\hline & Buena & $48(41,0 \%)$ & $26(50,0 \%)$ & $62(32,5 \%)$ & $34(41,5 \%)$ \\
\hline & Regular & $34(29,1 \%)$ & $7(13,5 \%)$ & $71(37,2 \%)$ & $23(28,0 \%)$ \\
\hline & Mala & $10(8,5 \%)$ & $2(3,8 \%)$ & $23(12,0 \%)$ & $2(2,4 \%)$ \\
\hline & $\mathrm{Ns} / \mathrm{Nr}$ & $3(2,6 \%)$ & $1(1,9 \%)$ & $0(0 \%)$ & $1(1,2 \%)$ \\
\hline & Total & $117(100 \%)$ & $52(100 \%)$ & $191(100 \%)$ & $82(100 \%)$ \\
\hline \multirow{4}{*}{$\begin{array}{l}\text { ¿Alguna vez le diagnosticaron } \\
\text { DIABETES? }\end{array}$} & $\mathrm{Si}$ & $27(23,1 \%)$ & $5(9,6 \%)$ & $43(22,5 \%)$ & $14(17,1 \%)$ \\
\hline & $\mathrm{No}$ & $87(74,4 \%)$ & $46(88,5 \%)$ & $146(73,4 \%)$ & $67(81,7 \%)$ \\
\hline & $\mathrm{Ns} / \mathrm{Nr}$ & $3(2,6 \%)$ & $1(1,9 \%)$ & $2(1,0 \%)$ & $1(1,2 \%)$ \\
\hline & Total & $117(100 \%)$ & $52(100 \%)$ & $191(100 \%)$ & $82(100 \%)$ \\
\hline \multirow{4}{*}{$\begin{array}{l}\text { ¿Alguna vez le diagnosticaron } \\
\text { HIPERTENSION? }\end{array}$} & $\mathrm{Si}$ & $64(54,7 \%)$ & $27(51,9 \%)$ & $131(68,6 \%)$ & $38(43,6 \%)$ \\
\hline & No & $50(42,7 \%)$ & $24(46,2 \%)$ & $57(29,8 \%)$ & $43(52,4 \%)$ \\
\hline & $\mathrm{Ns} / \mathrm{Nr}$ & $3(2,6 \%)$ & $1(1,9 \%)$ & $3(1,6 \%)$ & $1(1,2 \%)$ \\
\hline & Total & $117(100 \%)$ & $52(100 \%)$ & $191(100 \%)$ & $82(100 \%)$ \\
\hline \multirow{4}{*}{$\begin{array}{l}\text { ¿Alguna vez le diagnosticaron } \\
\text { ENFERMEDADES DEL CORAZÓN? }\end{array}$} & $\mathrm{Si}$ & $25(21,4 \%)$ & $13(25,0 \%)$ & $64(33,5 \%)$ & $18(22,0 \%)$ \\
\hline & No & $89(76,1 \%)$ & $38(73,1 \%)$ & $125(64,5 \%)$ & $62(75,6 \%)$ \\
\hline & $\mathrm{Ns} / \mathrm{Nr}$ & $3(2,6 \%)$ & $1(1,9 \%)$ & $2(1,2 \%)$ & $2(2,4 \%)$ \\
\hline & Total & $117(100 \%)$ & $52(100 \%)$ & $191(100 \%)$ & $82(100 \%)$ \\
\hline \multirow{4}{*}{$\begin{array}{l}\text { ¿Alguna vez le diagnosticaron } \\
\text { ACCIDENTE CEREBRO VASCULAR? }\end{array}$} & $\mathrm{Si}$ & $9(7,7 \%)$ & $1(1,9 \%)$ & $9(4,7 \%)$ & $4(4,9 \%)$ \\
\hline & No & $104(88,9 \%)$ & $50(96,2 \%)$ & $180(94,2 \%)$ & $77(93,9 \%)$ \\
\hline & $\mathrm{Ns} / \mathrm{Nr}$ & $4(3,4 \%)$ & $1(1,9 \%)$ & $2(1,0 \%)$ & $1(1,2 \%)$ \\
\hline & Total & $117(100 \%)$ & $52(100 \%)$ & $191(100 \%)$ & $82(100 \%)$ \\
\hline \multirow{4}{*}{$\begin{array}{l}\text { ¿Alguna vez le diagnosticaron TUMOR } \\
\text { MALIGNO? }\end{array}$} & $\mathrm{Si}$ & $2(1,7 \%)$ & $7(13,5 \%)$ & $11(5,8 \%)$ & $5(6,1 \%)$ \\
\hline & No & $112(95,7 \%)$ & $43(87,2 \%)$ & $178(93,2 \%)$ & $75(95,1 \%)$ \\
\hline & $\mathrm{Ns} / \mathrm{Nr}$ & $3(2,6 \%)$ & $2(3,8 \%)$ & $2(1,0 \%)$ & $2(2,4 \%)$ \\
\hline & Total & $117(100 \%)$ & $52(100 \%)$ & $191(100 \%)$ & $82(100 \%)$ \\
\hline \multirow{4}{*}{$\begin{array}{l}\text { ¿Alguna vez le diagnosticaron } \\
\text { ARTRITIS/ARTROSIS/ } \\
\text { REUMATISMO? }\end{array}$} & $\mathrm{Si}$ & $37(31,6 \%)$ & $20(38,5 \%)$ & $114(59,7 \%)$ & $52(63,4 \%)$ \\
\hline & No & $75(64,1 \%)$ & $31(59,6 \%)$ & $74(38,7 \%)$ & $28(34,1 \%)$ \\
\hline & $\mathrm{Ns} / \mathrm{Nr}$ & $5(4,3 \%)$ & $1(1,9 \%)$ & $3(1,6 \%)$ & $2(2,4 \%)$ \\
\hline & Total & $117(100 \%)$ & $52(100 \%)$ & $191(100 \%)$ & $82(100 \%)$ \\
\hline
\end{tabular}

$\mathrm{CSPC}=$ Comedores Sociales de la Provincia de Córdoba; DASPU = Dirección de Asistencia Social del Personal Universitario. Ns/Nr= No sabe/No responde. 
En relación al autoinforme de enfermedades crónicas, en la tabla 4 se indican la prevalencias de las mismas. Se observó que en hombres del grupo CSPC existió una mayor prevalencia de diabetes $(\mathrm{OR}=2,9 \mathrm{IC}=1,3-7,9 \mathrm{p}=0,027)$, mientras que existió menor prevalencia de tumor maligno $(\mathrm{OR}=0,1 \mathrm{IC}=0,02-0,5 \mathrm{p}=0,004)$. El resto de las enfermedades no presentaron diferencias con significancia estadística.

Finalmente, las mujeres provenientes del grupo CSPC presentaron mayor riesgo de hipertensión $\quad(\mathrm{OR}=2,6 \quad \mathrm{IC}=1,5-4,4 \quad \mathrm{p}=0,001)$ en relación a las mujeres del grupo DASPU; mientras que en el resto de las enfermedades no presentaron diferencias estadísticamente significativas.

\section{DISCUSIÓN}

En el presente trabajo se analizan las diferencias en el estado de salud y nutrición en dos colectivos de adultos mayores de diferente nivel educativo y de ingresos.

En primer lugar se observaron diferencias en los indicadores antropométricos de los adultos mayores de sexo masculino, según pertenencia (CSPC o DASPU); mientras que en las mujeres no se encontraron mayores diferencias entre ambas instituciones, salvo en mayor talla, menor circunferencia de cadera y menor IMC en mujeres del grupo DASPU. En el caso particular de la talla, la ecuación de Chumlea ${ }^{18}$ estimó mejor la talla en el grupo DASPU en ambos sexos, lo cual podría estar relacionado con numerosos factores; según algunos autores: la historia nutricional, el estilo de vida, el ambiente y las diferencias étnicas son variables que influencian las proporcionalidades de los segmentos corporales, lo cual afecta la capacidad de predicción de las ecuaciones. ${ }^{19,20}$
Respecto a los indicadores del estado nutricional en mujeres y hombres, se observa, como en otros estudios, ${ }^{21-24}$ una alta proporción de obesidad en mujeres (pero no en hombres) provenientes de sectores de bajo nivel educativo y/o bajos ingresos, asociado a un aumento de la obesidad abdominal. Existen diversas hipótesis en relación a este hecho, la más fuerte postula que el menor consumo de alimentos de alto costo y calidad nutricional en los hogares de bajos ingresos, suele afectar más a las mujeres, debido a que muchas veces modifican su ingesta para proteger a otros miembros de la familia..$^{25,26}$

Por otra parte, la mitad de la población de mujeres y hombres del grupo DASPU presentaron malnutrición por exceso; mientras que los hombres del grupo CSPC, a diferencia de las mujeres, presentaron una mayor proporción de malnutrición por déficit, lo cual conlleva un mayor riesgo de morbilidad y mortalidad en este grupo. ${ }^{4,27}$ A nivel global, los resultados en relación a la malnutrición tanto por déficit como por exceso en ambos grupos y sexos, refleja la necesidad de implementar medidas tendientes a favorecer una adecuada nutrición en este grupo etario, como la promoción de hábitos alimentarios saludables a través de la educación alimentaria nutricional, el fomento de la actividad física; así como medidas que favorezcan la seguridad alimentaria en los adultos mayores más desfavorecidos, desde el punto de vista social y económico. $3,7,27$

En relación a la percepción del estado de salud, el grupo DASPU, conformado por adultos mayores de altos niveles de escolarización y mayores ingresos que el grupo CSPC; presentó una mayor apreciación positiva de su estado de salud. Esto coincide con otras investigaciones realizadas en adultos ${ }^{28-30}$ en donde la percepción del estado de salud fue mejor en aquellos con alto nivel de instrucción o ingresos más elevados. Estas diferencias parecen estar fundamentadas 
en que altos niveles sociales utilizan un mayor número de variables cuando evalúan su salud, incluyendo aspectos como estar en forma y activos físicamente, además de la ausencia de enfermedad, y otros aspectos del bienestar, como la felicidad.30 Por otra parte, al igual que en otros estudios, ${ }^{27-29}$ se observó que los hombres perciben mejor su salud que las mujeres. Esto último puede deberse a que las mujeres en general sufren las consecuencias de mayores incapacidades a edades más avanzadas. ${ }^{31}$

Uno de los resultados más significativos son las diferencias que se observan en las prevalencias de enfermedades entre los grupos, lo cual estaría indicando el mayor riesgo de padecer algunas afecciones según los niveles de instrucción y económico. Revisando las principales patologías, puede mencionarse lo relativo a tumores, diabetes e hipertensión arterial. Los tumores malignos fueron más prevalentes en el grupo de hombres DASPU. Algunos tipos de cáncer, como el cáncer de pulmón, están relacionados a ciertas conductas individuales, como el consumo de tabaco, las cuales difieren según el nivel socioeconómico. ${ }^{32}$ En el caso de la diabetes y de la hipertensión arterial, se observaron mayores prevalencias en varones y mujeres de CSPC, respectivamente. Es decir, que en los hombres de CSPC -a pesar que posee menor frecuencia de malnutrición por exceso- presentan más riesgo de padecer diabetes. En el caso de las mujeres, a pesar que ambos grupos presentaron similares prevalencias de estado nutricional, las de CSPC presentaron mayor riesgo de padecer hipertensión arterial. Esta paradoja puede estar explicada por la hipótesis de que las deficiencias nutricionales en la niñez se relacionan con el riesgo a padecer enfermedades crónicas no transmisibles en la adultez. ${ }^{33,34}$ Sin embargo, para corroborar esta hipótesis se requieren de estudios que evalúen tanto las condiciones actuales de los adultos mayores como las condiciones de la vida en la niñez. Otra hipótesis, se relaciona con los hábitos de los adultos mayores; ya que en otros estudios se ha observado que un bajo nivel educativo se asocia con una menor adherencia a una dieta saludable, así como una menor realización de actividad física. ${ }^{35,36}$

Las principales limitaciones del presente estudio se relacionan con su carácter transversal, lo cual no permite establecer asociaciones causa y efecto. Por otra parte, no se pueden generalizar los datos a la totalidad de la ciudad de Córdoba, ya que no se trató de una muestra representativa, sino intencional. Sin embargo, sí se pueden generalizar los datos a cada grupo (CSPC, DASPU) estudiado.

\section{CONCLUSIONES}

Como principal conclusión se observa que las condiciones sociales de vida influyen en el estado nutricional y en el estado de salud de los adultos mayores, implicando riesgos diferentes para ambos grupos.

Estos datos son de importancia para definir criterios de acción en los grupos de ancianos analizados. Por una parte, la elevada prevalencia de malnutrición por exceso en ambas poblaciones requiere de medidas universales, relacionadas con la promoción de hábitos de vida saludables, que incluya el fomento de una alimentación adecuada y la actividad física en este grupo poblacional. Por otra parte, este trabajo también refleja las inequidades en relación a las condiciones de vida de los adultos mayores, que deben ser abordadas a través de acciones enfocadas a los grupos sociales vulnerables.

Diseñar políticas para atacar estos problemas se vuelve aún más importante si se considera el proceso de envejecimiento poblacional actual y futuro al que se enfrentará tanto Córdoba como Argentina. Así, este incremento porcentual de los adultos mayores respecto del total poblacional implica el desarrollo de instrumentos que den soporte no sólo a la salud física si no también en los otros ámbitos de la vida de forma tal que propicien el bienestar de los mayores. 


\section{REFERENCIAS}

1. Huenchuan S. Envejecimiento, derechos humanos y políticas públicas. Santiago de Chile: CEPAL; 2009.

2. Comisión Económica para América Latina y el Caribe (CEPAL). Transformaciones demográficas y su influencia en el desarrollo de América Latina y el Caribe. Santiago de Chile: CEPAL; 2008.

3. Woo J. Obesity in older persons. Curr Opin Clin Nutr Metab Care 2015;18(1):5-10.

4. Al Snih S, Graham JE, Kuo YF, Goodwin JS, Markides KS, Ottenbacher K. Obesity and disability: relation among older adults living in Latin America and the Caribbean. Am J Epidemiol 2010;171(12):1282-8.

5. Chatterji S, Byles J, Cutler D, Seeman T, Verdes E. Health, functioning and disability in older adults-present status and future implications. Lancet [Internet] 2014 [acesso em 19 nov 2014]; 385(9967):481-576. Disponível em: http://www. thelancet.com/journals/lancet/article/PIIS01406736\%2814\%2961462-8/abstract.

6. Prince MJ, Wu F, Guo Y, Gutierrez Robledo LM, O'Donnell M, Sullivan R, et al. The Burden of disease in older people and implications for health policy and practice. Lancet [Internet] 2014 [acesso em 19 nov 2014];385(9967):549-62. Disponível em: http://www.thelancet.com/journals/lancet/article/ PIIS0140-6736\%2814\%2961347-7/abstract.

7. Vagetti GC, Barbosa VC Filho, Moreira NB, Oliveira VD, Mazzardo O, Campos WD. Association between physical activity and quality of life in the elderly: a systematic review, 2000-2012. Rev Bras Psiquiatr 2014;36(1):76-88.

8. Dy SM, Pfoh ER, Salive ME, Boyd CM. Healthrelated quality of life and functional status quality indicators for older persons with multiple chronic conditions. J Am Geriatr Soc 2013;61(12):2120-7.

9. Karlsdotter K, Martín Martín JJ, Gonzalez MPL. Influencia de La renta, la desigualdad de renta y el capital social en la salud de los mayores de 65 años en España en 2007. Gac Sanit 2011;25 Supl 2:59-65.

10. Popkin BM. Contemporary nutritional transition: determinants of diet and its impact on body composition. Proc Nutr Soc 2011;70(1):82-91.

11. Price MJ, Ebrahim S, Acosta D, Ferri CP, Guerra M, Huang Y, et al. Hypertension prevalence, awareness, treatment and control among older people in Latin America, India and China: a 10/66 cross-sectional population-based survey. J Hypertens 2012;30(1):177-87.
12. Albala C, Lebrao ML, León Díaz EM, Ham-Chande R, Hennis AJ, Palloni A, et al. Encuesta Salud Bienestar y Envejecimiento (SABE): Metodología de La encuesta y perfil de la población estudiada. Rev Panam Salud Pública 2005;17(5/6):307-22.

13. Elgart J, Pfirter G, Gonzalez L, Caporale J, Cormillot A, Chiappe Ml, et al. Obesidad en Argentina: epidemiología, morbimortalidad e impacto económico. Rev Argent Salud Pública 2010;1(5):6-12.

14. Ministerio de Salud de la Nación. Encuesta Nacional de Factores de Riesgo:documento de resultados. Buenos Aires: Ministerio de Salud de la Nación; 2006.

15. Ferrante D, Linetzky B, Konfino J, King A, Virgolini M, Laspiur S. Encuesta Nacional de Factores de Riesgo 2009: evolución de la epidemia de enfermedades crónicas no transmisibles en Argentina. Estudio de corte transversal. Rev Argent Salud Publica 2011;2(6):34-41.

16. Quételet A. Anthropométrie ou mesure des différentes facultés de l'homme. Bruxelles: C. Muquardt; 1870.

17. Organización Panamericana de la Salud. Guía Clínica para la atención primaria a las personas adultas mayores. Washington, DC: OPS/OMS; 2002. Parte I, Módulo 5, Valoración nutricional del adulto mayor. p. 57-70. (Serie Materiales de Capacitación no 1.)

18. Chumlea WC, Roche AF, Steinbaugh ML. Estimating stature from knee height for persons 60 to 90 years of age. J Am Geriatr Soc 1985;33(2):116-20.

19. Barceló M, Torres O, Mascaró J, Francia E, Cardona D, Ruiz D. Assessing Nutritional status in the elderly; evaluation of Chumlea's equations for weight. Nutr Hosp 2013;28(2):314-18.

20. Guzmán Hernández C, Reinoza Calderón G, Hernández Hernández RA. Estimación de la estatura a partir de la longitud de pierna medida con cinta métrica. Nutr Hosp 2005;20(5):358-63.

21. Schlüssel MM, Silva AA, Pérez-Escamilla R, Kac G. Household food insecurity and excess weight/obesity among Brazilian women and children: a life course approach. Cad Saúde Pública 2013;29(2):219-26.

22. Martin MA, Lippert AM. Feeding her children, but risking her health: the intersection of gender, household food insecurity and obesity. Soc Sci Med 2012;74(11):1754-64. 
23. Shariff ZM, Sulaiman N, Jalil RA, Yen WC, Yaw $\mathrm{YH}$, Taib MN, et al. Food insecurity and the metabolic syndrome among women from low income communities in Malaysia. Asia Pac J Clin Nutr 2014;23(1):138-47.

24. Boissonnet C, Schargrodsky H, Pellegrini F, Macchia A, Marcet Champagne B, Wilson E. Educational inequalities in obesity, abdominal obesity, and metabolic syndrome in seven Latin American cities: the CARMELA Study. Eur J Cardiovasc Prev Rehabil 2011;18:550-6.

25. Martín-Fernández J, Caillavet F, Lhuissier A, Chauvin P. Food insecurity, a determinant of obesity?: an analysis from a population-based survey in the Paris metropolitan area, 2010. Obes Facts 2014;7(2):120-9.

26. Franklin B, Jones A, Love D, Puckett S, Macklin J, White-Means S. Exploring mediators of food insecurity and obesity: a review of recent literature. J Community health 2012;37(1):253-65.

27. Jahangir E, De Schutter A, Lavie CJ. Low weight and overweightness in older adults: risk and clinical management. Prog Cardiovasc Dis 2014;57(2):127-33.

28. López E, Findling L, Abramzón M. Desigualdades en Salud: ¿es diferente la percepción de morbilidad de varones y mujeres? Salud Colectiva 2006;2(1):61-74.

29. Kaleta D, Polańska K, Dziankowska-Zaborszczyk E, Hanke W, Drygas W. Factors influencing selfperception of health status. Cent Eur J Public Health 2009;17(3):122-7.
30. Maniecka-Bryla I, Gajewska O, Burzynka M, Bryla M. Factors associated with self-rathed health $(\mathrm{SRH})$ of a University of the Third Age (U3A) class participants. Arch Gerontol Geriatr 2013;57(2):156-61.

31. Crimmins EM, Kim JK, Solé-Auró A. Gender differences in health: results from SHARE, ELSA and HRS. Eur J Public Health 2011;21(1):89-91.

32. Merletti F, Galassi C, Spadea T. The socioeconomic determinants of cancer. Environ Health 2011;10 Supl 1:S2-7.

33. Fall CH. Fetal malnutrition and long-term outcomes. In: Bhatia Jatinder, Bhutta ZA, Kalhan SC, editors. Maternal and child nutrition: the first 1,000 Days: Proceedings of the 74th Nestle Nutrition Institute Workshop ;11-14 mar 2012;[s.1.]: Nestlé Nutrition Institute Workshop Series; 2013. p.11-25.

34. Langley-Evans SC, McMullen S. Developmental origins of adult disease. Med Princ Pract 2010;19(2):87-98.

35. Katsarou A, Tyrovolas S, Psaltopoulou T, Zeimbekis A, Tsakountakis N, Bountziouka V, et al. Socioeconomic status, place of residence and dietary habits among the elderly: the Mediterranean islands study. Public Health Nutr 2010;13(10):1614-21.

36. Hu EA, Toledo E, Diez-Espino J, Estruch R, Corella D, Salas-Salvado J, et al. Lifestyles and Risk Factors Associated with Adherence to the Mediterranean Diet: a baseline assessment of the PREDIMED trial. PLos ONE 2013;8(4):e60166. 